\title{
Soluble E-cadherin and IL-6 serum levels in patients affected by prostate cancer before and after prostatectomy
}

\author{
FORTUNATA IACOPINO ${ }^{1}$, FRANCESCO PINTO $^{2}$, ALESSANDRO BERTACCINI $^{3}$, \\ ALESSANDRO CALARCO $^{2}$, GABRIELLA PROIETTI $^{1}$, ANGELO TOTARO $^{2}$, \\ GIUSEPPE MARTORANA ${ }^{3}$, PIERFRANCESCO BASSI ${ }^{2}$ and GIGLIOLA SICA ${ }^{1}$ \\ ${ }^{1}$ Institute of Histology and Embryology, Faculty of Medicine and ${ }^{2}$ Department of Urology, Faculty of Medicine, \\ Catholic University of the Sacred Heart, I-00168 Rome; ${ }^{3}$ Department of Urology, Sant'Orsola-Malpighi \\ Hospital, The Alma Mater Studiorum University of Bologna, Padiglione 1, I-40138 Bologna, Italy
}

Received January 31, 2012; Accepted March 27, 2012

DOI: $10.3892 /$ or.2012.1785

\begin{abstract}
Prostate specific antigen (PSA) is still the best available tumour marker in prostate cancer $(\mathrm{PCa})$, but presents some limits. Therefore, there is a need for novel markers in the detection and management of PCa. The $80-\mathrm{kDa}$ soluble form of E-cadherin (sE-cad) and the cytokine IL-6 are being discussed as supplemental serum markers for PCa. In this study, sE-cad and IL-6 serum levels were determined in patients with pathological localized or locally advanced $\mathrm{PCa}$ without any previous treatment. These patients underwent radical retropubic prostatectomy (RRP) in accordance with the EAU Guidelines on Prostate Cancer. The molecules were determined via immunoenzymatic assays in samples collected before and after surgery. Statistical analysis was performed by Student's t-test and Pearson's correlation test. sE-cad levels were $6.0 \pm 2.7$ and $4.6 \pm 2.3 \mu \mathrm{g} / \mathrm{ml}$, before and after RRP, respectively. A highly statistically significant decrease in sE-cad concentrations after RRP was observed $(\mathrm{P}<0.0001)$, in 50/61 patients $(82 \%)$. sE-cad levels before and after surgery were correlated (Pearson's correlation coefficient, $r=0.6993, \mathrm{P}<0.0001$ ). sE-cad values detected after surgery were higher in patients with PSA levels $>10 \mathrm{ng} / \mathrm{ml}(\mathrm{P}<0.05)$. sE-cad levels before RRP were significantly higher in patients with G3 tumours compared to those with G2 tumours $(P<0.02)$. Finally, sE-cad concentrations both before and after surgery were higher in tumours with Gleason score $=7$ compared to those with Gleason score $<7(\mathrm{P}<0.002$ and $\mathrm{P}<0.05$, respectively). Preliminary data from 20 patients indicated a statistically significant increase in IL-6 levels after RRP (11.2 vs. $7.2 \mathrm{pg} / \mathrm{ml}, \mathrm{P}<0.001)$. This is the first study on the reduction in sE-cad levels after RRP
\end{abstract}

Correspondence to: Professor Gigliola Sica, Institute of Histology and Embryology, Faculty of Medicine, Catholic University of the Sacred Heart, Largo Francesco Vito 1, I-00168 Rome, Italy E-mail: gigliola@rm.unicatt.it; ibiis@rm.unicatt.it

Key words: prostate cancer, serum markers, soluble E-cadherin, IL-6 in PCa patients. Moreover, it shows that preoperative sE-cad concentrations are higher in patients with less differentiated PCa. Promising findings of this pilot study may lead to investigation of sE-cad in a larger study with follow-up.

\section{Introduction}

Prostate-specific antigen (PSA) represents the most reliable tumour marker for the diagnosis and management of prostate cancer (PCa) established to date. Nevertheless, PSA presents some limitations including the fact that it does not distinguish indolent from aggressive cancers. This substantially results in the risk of overdiagnosis and overtreatment. Moreover, PSA does not provide prognostic information $(1,2)$. For these reasons, there is an urgent need for new markers to enhance the clinical management of PCa (3). Among biomarkers measured in the serum, adhesion molecules and inflammatory cytokines and their receptors are interesting candidates from a clinical point of view (4-7).

E-cadherin (E-cad) is a calcium-dependent transmembrane protein involved in maintaining cell polarity and normal epithelial structure (8). The loss of E-cad has been recognized as an important mechanism for cancer progression (9). Aberrant expression of the E-cad/catenin complex has been found in PCa and disruption of E-cad is associated with reduced survival $(10,11)$. E-cad ectodomain shedding determines the release into the body fluids of a soluble $80 \mathrm{kDaE}$-cad fragment (sE-cad) (12). High sE-cad serum levels are present in cancer patients when compared with those of healthy individuals and they are significantly associated with metastasis, recurrence, and prognosis in some malignancies, including PCa (13-18). It has been reported that sE-cad is involved in stimulation of invasion in a paracrine manner, in promotion of cell junction disruption and of cell dissemination thereby inducing the migratory phenotype (19). Moreover, the highest expression levels of $\mathrm{sE}$-cad have been found in patients with metastatic cancers, and high levels at the time of diagnosis are associated with a significant risk of biochemical failure (16-18). Nevertheless, there is no agreement among authors about correlation between sE-cad and clinical or pathological para-meters. Ahmed et al (18) found a relationship between 
sE-cad and PSA or poor Gleason grade, whereas other authors did not report any correlation between any clinical parameters and sE-cad levels (17).

Regarding interleukins (IL), they are pleiotropic cytokines with a wide range of biological activities including immune regulation, hematopoiesis, inflammation and oncogenesis (20). High levels of IL-6 in serum are associated with more voluminous tumours, higher Gleason score and the presence of lymph node metastases $(21,22)$. These levels are dramatically elevated in patients with PCa metastatic to bone (23). Plasma levels of IL- 6 and its soluble receptor in patients with clinically localized PCa are independent predictors of biochemical progression after surgery. The incorporation of the soluble receptor for IL-6 in standard predictive nomograms together with other biomarkers facilitates the identification of patients for adjuvant therapy after radical retropubic prostatectomy (RRP) (24).

In the current study, for the first time the evaluation of E-cad levels was performed in serum samples collected before and after RRP of patients affected by primary PCa. In a small cohort of the same group of patients, IL- 6 values were determined. The potential use of these molecules as serum markers for PCa is discussed.

\section{Patients and methods}

Patients. Sixty-one patients with clinically localized PCa, who underwent RRP, in accordance with the EAU Guidelines on Prostate Cancer, were enrolled into the study. None of the patients received preoperative hormonal treatment or radiotherapy. On the basis of the current pathological TNM classification, tumours were classified as local or locally advanced PCa. Patients underwent standard pelvic lymphadenectomy (external iliac and obturatory lymph nodes removal) if PSA $\geq 10 \mathrm{ng} / \mathrm{ml}$ and/or biopsy Gleason score $\geq 7$. Serum samples were collected with standard procedure before and after surgical treatment, at least three days after i.v. infusion of fluids in the post-operative period, and kept at $-80^{\circ} \mathrm{C}$ until the time of sE-cad and IL- 6 determinations. For the present analyses, samples were collected at a session between 7:00 and 9:00 a.m. All patients gave oral consent to use their serum samples for research purpose.

Serum analysis of $s E$-cad and IL-6. Serum concentrations of the $80-\mathrm{kDa}$ fragment were analysed using the Human E-cadherin EIA kit from Zymed Laboratories (San Francisco, CA, USA). In this commercially available kit, HECD-1 is the E-cad-specific antibody known to map to the extracellular domain of E-cad, from where the $80-\mathrm{kDa}$ fragment derives. The absorbance was measured at $450 \mathrm{~nm}$ using a standard 96-well microtiter plate reader.

IL-6 was determined by using a human IL-6 ELISA kit (Thermo Scientific, Rockford, IL, USA). The absorbance was measured at 450 and $550 \mathrm{~nm}$ using a standard 96-well microtiter plate reader. All samples were run in duplicate and the mean value was used for statistical analysis.

Serum samples collected from healthy donors were tested in each kit as internal control. The control group consisted of 11 healthy men under 30 years of age with no history of previous prostatic disease.
Table I. Patients and tumour characteristics.

Number of Patients

Age (years), median

Total PSA (ng/ml), mean \pm SD

$8.98 \pm 5.7$

Pathological T-stage (N)

T2

42

T3

Unknown

Gleason score $(\mathrm{N})$

$<7$

$=7$

$>7$

Unknown

1

Tumour grade $(\mathrm{N})$

G2

16

G3

G4

Unknown 15

Statistical analysis. Data analysis was performed by using the GraphPad Prism version 3. Normality test was used to determine whether data are modelled by a normal distribution. The differences between two groups were explored by paired Student's t-test. The relationship between sE-cad and IL-6 serum content and pathological variables was evaluated by linear regression analysis and Pearson's correlation test.

Patients were stratified into four groups according to the major prognostic factors: PSA, T-stage, grade and Gleason score. sE-cad and IL-6 levels in patient subgroups were analyzed using the unpaired Student's t-test. $\mathrm{P}<0.05$ was considered as statistically significant.

\section{Results}

sE-cad

Patients. The median age of the 61 patients was 67 years (range, 55-74). The mean preoperative PSA for the 61 patients was $8.98 \pm 5.7 \mathrm{ng} / \mathrm{ml}$ (range, 2.38-28.6). The pathological T-stage was T2 in 42 patients, T3 in 15 patients and unknown in 4 patients. Pathological Gleason score was $<7$ in 25 cases, $=7$ in 31 cases, $>7$ in 4 cases and unknown in 1 case. Tumour grade was G2 in 16 cases, G3 in 27 cases, G4 in 3 cases, unknown in 15 cases (Table I).

Of the 61 patients, 44 had a bilateral neoplasia, 17 had positive surgical margin status, 30 had perineural and 2 seminal vesicle infiltration. Mean wet weight of prostate was $53.3 \pm 33.5 \mathrm{~g}$.

sE-cad serum levels. The sE-cad was detectable and measured in all serum samples of the $61 \mathrm{PCa}$ patients. The mean $\pm \mathrm{SD}$ of $\mathrm{sE}$-cad value was $6.01 \pm 2.7 \mu \mathrm{g} / \mathrm{ml}(95 \% \mathrm{CI}$, 5.32-6.70) in the samples collected before RRP (pre-surgery) and $4.62 \pm 2.3 \mu \mathrm{g} / \mathrm{ml}(95 \% \mathrm{CI}, 4.01-5.21)$ in those obtained after RRP (post-surgery) (Fig. 1). Serum E-cad levels were signifi- 


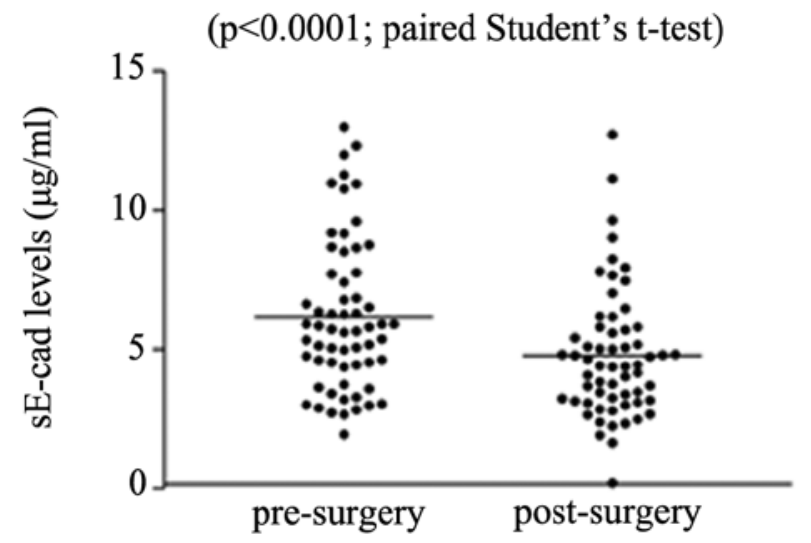

Figure 1. Soluble E-cadherin (sE-cad) levels determined in serum samples collected before and after prostatectomy. Mean values are indicated by horizontal bars.

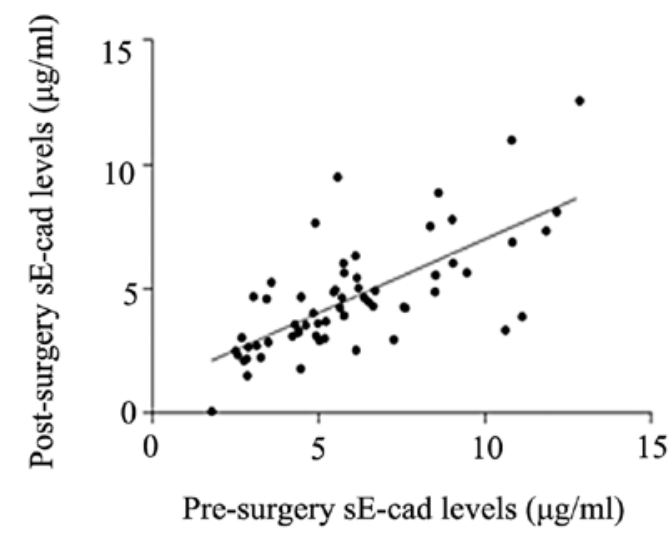

Pearson's correlation coefficient, $\mathrm{r}=0.6993$ $\mathrm{p}<0.0001$

Figure 2. Relationship between soluble E-cadherin (sE-cad) levels determined in serum samples collected before and after prostatectomy determined by using Pearson's Correlation test.

cantly higher than those of healthy subjects $(4.09 \pm 0.45 \mu \mathrm{g} / \mathrm{ml}$, 95\% CI, 3.09-5.09, $\mathrm{n}=11, \mathrm{P}=0.025)$.

A statistically significant $(\mathrm{P}<0.0001)$ reduction in $\mathrm{sE}$-cad levels was observed between the samples collected after surgery and those collected before surgery. It should be noted that $\mathrm{sE}$-cad values were lower in 50/61 cases (82\%) after RRP. In $34 / 50$ cases $(68 \%)$, the decrease was $>20 \%$. In $11 / 61(18 \%)$ patients, an increase in sE-cad levels was found and in $5 / 11$ cases $(45 \%)$ this increase was $>20 \%$. There was no statistically significant difference in $\mathrm{sE-cad}$ values detected in serum samples taken after surgery and those obtained from healthy donors.

A positive correlation between sE-cad values before and after RRP (Pearson correlation coefficient, $r=0.67, \mathrm{P}<0.0001$ ) was found (Fig. 2). On the other hand, no correlation was observed between sE-cad levels and any of the following parameters: preoperative PSA level, tumour stage (TNM), Gleason score, grade, surgical margin status, perineural invasion and weight of prostate gland (data not shown). sE-cad values were retrospectively stratified into three groups based

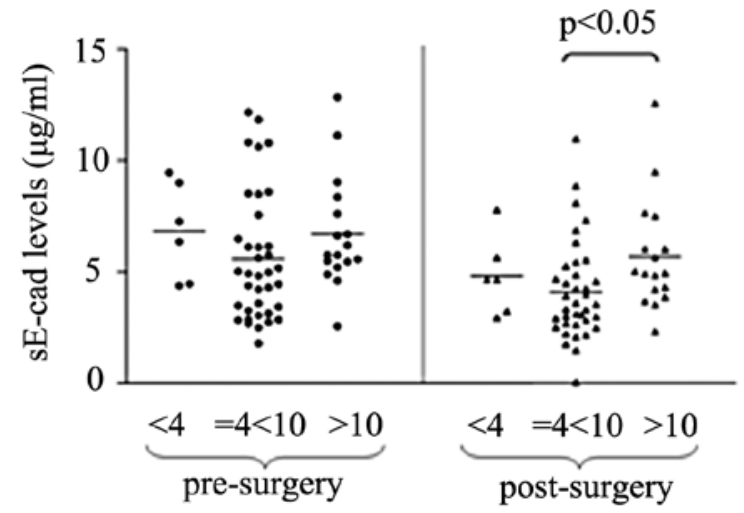

Figure 3. Soluble E-cadherin (sE-cad) levels determined in serum samples collected before and after prostatectomy. Values were stratified into 3 groups based upon PSA levels $(<4,=4<10,>10 \mathrm{ng} / \mathrm{ml})$. Mean values are indicated by horizontal bars.

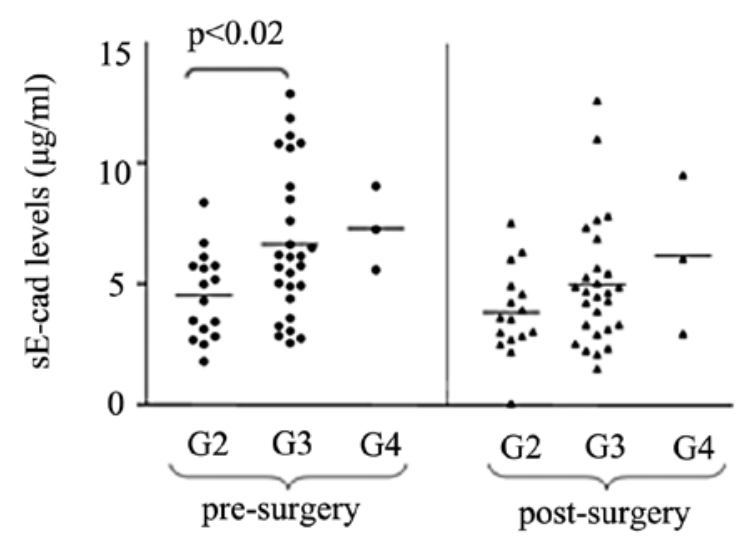

Figure 4. Soluble E-cadherin (sE-cad) levels determined in serum samples collected before and after prostatectomy. Values were stratified into 3 groups based upon tumour grade (G2, G3 and G4). Mean values are indicated by horizontal bars.

upon PSA levels, Gleason score, grade and T-stage. This analysis revealed that $\mathrm{sE}$-cad values detected after surgery were significantly higher in patients with PSA levels $>10 \mathrm{ng} / \mathrm{ml}$ $(\mathrm{P}<0.05$, Student's t-test, Fig. 3). sE-cad levels obtained before RRP were significantly higher in patients with G3 tumours than in those with $\mathrm{G} 2$ tumours $(\mathrm{P}<0.02$, Student's t-test, Fig. 4) and $\mathrm{sE}$-cad concentrations both before and after surgery were higher in tumours with Gleason score $=7$ than in those with Gleason score $<7(\mathrm{P}<0.002$ and $\mathrm{P}<0.05$, respectively, Student's t-test, Fig. 5). No statistically significant difference in the levels of sE-cad was found in groups of patients with different T-stage.

IL-6

Patients. IL-6 levels were determined in 20 patients randomly chosen. The median age of these patients was 67 years (range, 57-72). The mean preoperative PSA for all 20 patients was $8.02 \pm 5.8 \mathrm{ng} / \mathrm{ml}$ (range, 2.8-28.6). The pathological T-stage was $\mathrm{T} 2$ in 11 patients, $\mathrm{T} 3$ in 5 patients and unknown in 4 patients. Gleason score was $<7$ in 4 cases; equal to 7 in 14 cases and $>7$ in 2 cases. Tumour grade was G2 in 2 cases, G3 in 10 cases, and unknown in 8 cases (Table II). 


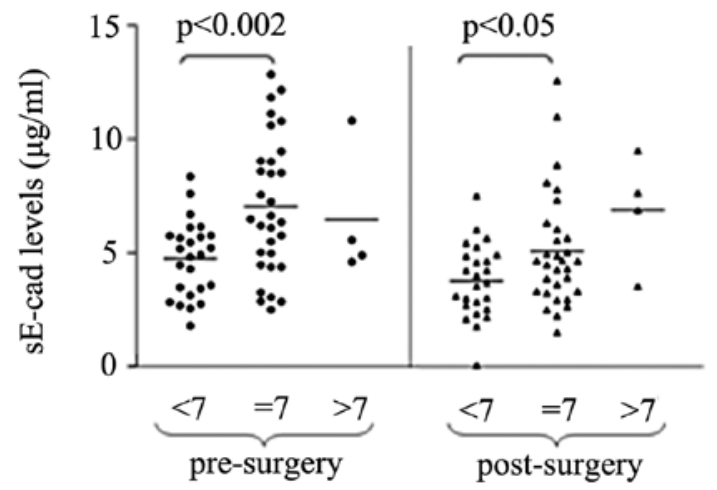

Figure 5. Soluble E-cadherin (sE-cad) levels determined in serum samples collected before and after prostatectomy. Values were stratified into 3 groups based upon Gleason score $(\leq 6,=7, \geq 8)$. Mean values are indicated by horizontal bars.

Table II. Patients and tumour characteristics of the cases in which IL-6 was evaluated.

Number of Patients

Age (years), median

Total PSA (ng/ml), mean \pm SD

Pathological T-stage (N)

$\mathrm{T} 2$

11

T3

5

Unknown

Gleason score $(\mathrm{N})$

$<7$

$=7$

$>7$

Tumour grade $(\mathrm{N})$

G2

G3

Unknown

Of the 20 patients, 16 had a bilateral neoplasia, 7 had positive surgical margin status, 13 had perineural and 1 seminal vesicle infiltration. The mean wet weight of prostate was $57.8 \pm 32.49 \mathrm{~g}$.

IL-6 serum levels. The mean \pm SD of IL-6 value was $7.19 \pm 2.29 \mathrm{pg} / \mathrm{ml}(95 \% \mathrm{CI}, 6.07-7.58)$ and $11.22 \pm 5.08 \mathrm{pg} / \mathrm{ml}(95 \%$ CI, 8.49-13.23) before and after RRP, respectively (Fig. 6). A statistically significant $(\mathrm{P}<0.001)$ increase in IL-6 levels was observed in the samples collected after surgery with respect to those collected before.

No correlation was found between IL-6 values and all the clinical and pathological parameters, or between IL-6 and sE-cad levels (data not shown).

\section{Discussion}

In the present study, the serum levels of sE-cad and IL- 6 were determined in samples collected before and after RRP from

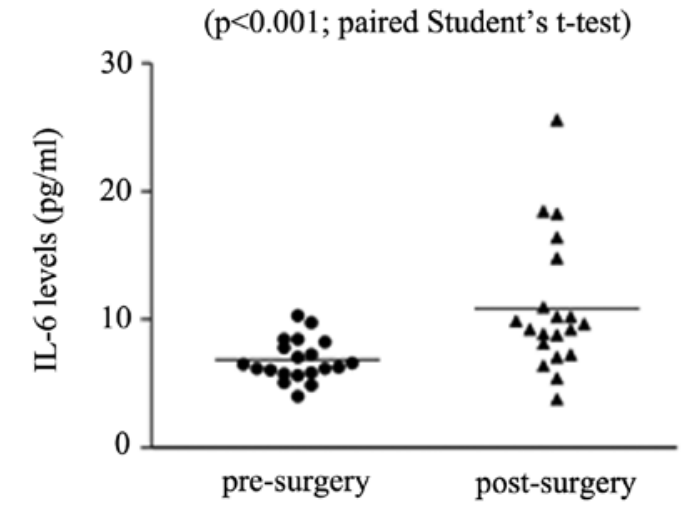

Figure 6. IL-6 levels determined in serum samples collected before and after prostatectomy. Mean values are indicated by horizontal bars.

a strictly selected group of patients affected by primary PCa. Our patients were homogeneous in age and had not received any previous treatment. This is quite important because it is reported in the literature that sE-cad and/or IL-6 values can be affected by age, treatment or circadian rhythm (25-27), even if there is no agreement among all authors particularly regarding sE-cad $(25,28)$. Moreover, as described in Patients and methods, blood samples were taken at least three days after i.v. infusion of fluids in the postoperative period to avoid less concentrations of blood solutes resulting from plasma dilution.

$\mathrm{sE}-\mathrm{cad}$ levels found in preoperative serum samples are in the range reported in the literature in patients affected by this or other types of cancers $(5,13,15,16,28)$. Values determined in patients were significantly higher than those observed in healthy subjects according to reports by other authors $(5,15,16,18,28)$. After RRP, a significant decrease in E-cad levels was observed. It should be pointed out that this was evident in 50/61 cases (82\%). To our knowledge, this is the first time that the variation in sE-cad values after RRP has been evaluated. For the first time, we have demonstrated that surgery diminishes the circulating form of E-cad in patients affected by PCa. The results from linear regression analysis and the Pearson's correlation coefficient indicate a relationship between E-cad levels in preoperative and postoperative samples. No correlation exists between levels of sE-cad and biochemical and histopathological findings. However, some interesting results were observed when patients were stratified into subgroups even if it should be pointed out that the limit of these subgroups is represented by the small number of subjects included in each of them. Despite this problem, it should be remarked that sE-cad levels were higher in patients with high grade tumours (G3 vs. G2 and Gleason score $=7$ vs. $<7$ ). This is in agreement with data from other authors (16-18), although we did not find any statistically significant correlation.

Moreover, in patients with PSA $=4<10 \mathrm{ng} / \mathrm{ml}$ the reduction in sE-cad after surgery was higher than that of patients with PSA $>10 \mathrm{ng} / \mathrm{ml}$.

Concerning IL-6, values before surgery were in the range reported in the literature $(22,24)$ and a statistically significant increase in IL-6 levels was observed after surgery. This could be consistent with the existence of inflammatory and regenerative phenomena intervening in the postoperative period. No 
correlation was found between the levels of IL- 6 and sE-cad detected before and after surgery in the same patients. As far as we know, there is no agreement in the literature concerning variation of serum IL-6 levels after surgery. This may be due to the differences in the time in which serum samples were collected. Shariat et al (22) found a decrease while Jurczok et al (29) observed an increase in IL-6 levels after RRP but in the first study samples were collected after 6-8 weeks while in the second after $0-72 \mathrm{~h}$ from the surgery.

In our study, there was no association between IL-6 serum levels and any of the clinical and pathological parameters considered.

Our findings indicate that surgery results in a decrease of sE-cad and in an increase in serum levels of IL-6, in patients affected by PCa. The same biological responses have been reported by Lin et al in patients affected by colon cancer who underwent surgery (30). Demonstration that the levels of these molecules are modified significantly in the post-surgery period must be considered important, particularly in view of their use as follow-up markers. It would be useful to compare the values of these markers measured during follow-up with the values determined before surgery or after surgery and this is what we are doing with a new cohort of patients.

At present, the small number of cases in which the cytokine IL-6 was determined does not allow us to draw any conclusion. As far as sE-cad is concerned, the overall results obtained so far, even if preliminary, are encouraging in the use of this molecule as a valuable marker associated with PSA in PCa diagnosis and as a prognostic marker at diagnosis. Further investigations with a larger number of subjects and a follow-up may better define the role of these molecules in the clinical management of patients with $\mathrm{PCa}$.

\section{References}

1. Strope SA and Andriole GL: Prostate cancer screening: current status and future perspectives. Nat Rev Urol 7: 487-493, 2010.

2. Daskivich TJ, Chamie K, Kwan L, Labo J, Palvolgyi R, Dash A Greenfield S and Litwin MS: Overtreatment of men with low-risk prostate cancer and significant comorbidity. Cancer 117: 2058-2066, 2011.

3. Steuber T, O'Brien MF and Lilja H: Serum markers for prostate cancer: a rational approach to the literature. Eur Urol 54: 31-40, 2008.

4. Beachy SH and Repasky EA: Using extracellular biomarkers for monitoring efficacy of therapeutics in cancer patients: an update. Cancer Immunol Immunother 57: 759-775, 2008.

5. De Wever O, Derycke L, Hendrix A, De Meerleer G, Godeau F, Depypere $\mathrm{H}$ and Bracke M: Soluble cadherins as cancer biomarkers. Clin Exp Metastasis 24: 685-697, 2007.

6. Pontes-Junior J, Reis ST, Dall'Oglio M, Neves de Oliveira LC, Cury J, Carvalho PA, Ribeiro-Filho LA, Moreira Leite KR and Srougi M: Evaluation of the expression of integrins and cell adhesion molecules through tissue microarray in lymph node metastases of prostate cancer. J Carcinog 8: 3, 2009.

7. Waugh DJ, Wilson C, Seaton A and Maxwell PJ: Multi-faceted roles for CXC-chemokines in prostate cancer progression. Front Biosci 13: 4595-4604, 2008.

8. Cavallaro U and Dejana E: Adhesion molecule signalling: not always a sticky business. Nat Rev Mol Cell Biol 12: 189-197, 2011 .

9. Jeanes A, Gottardi CJ and Yap AS: Cadherins and cancer: how does cadherin dysfunction promote tumor progression? Oncogene 27: 6920-6929, 2008.

10. Jaggi M, Johansson SL, Baker JJ, Smith LM, Galich A and Balaji KC: Aberrant expression of E-cadherin and betacatenin in human prostate cancer. Urol Oncol 23: 402-406, 2005 .
11. Musial J, Sporny S and Nowicki A: Prognostic significance of E-cadherin and ezrin immunohistochemical expression in prostate cancer. Pol J Pathol 58: 235-243, 2007.

12. van Kilsdonk JW, van Kempen LC, van Muijen GN, Ruiter DJ and Swart GW: Soluble adhesion molecules in human cancers: sources and fates. Eur J Cell Biol 89: 415-427, 2010.

13. Weiss JV, Klein-Scory S, Kübler S, Reinacher-Schick A, Stricker I, Schmiegel W and Schwarte-Waldhoff I: Soluble E-cadherin as a serum biomarker candidate: elevated levels in patients with late-stage colorectal carcinoma and FAP. Int J Cancer 128: 1384-1392, 2011.

14. Chung Y, Law S, Kwong DL and Luk JM: Serum soluble E-cadherin is a potential prognostic marker in esophageal squamous cell carcinoma. Dis Esophagus 24: 49-55, 2011

15. Gogali A, Charalabopoulos K, Zampira I, Konstantinidis AK, Tachmazoglou F, Daskalopoulos G, Constantopoulos SH and Dalavanga Y: Soluble adhesion molecules E-cadherin, intercellular adhesion molecule-1, and E-selectin as lung cancer biomarkers. Chest 138: 1173-1179, 2010.

16. Kuefer R, Hofer MD, Zorn CS, Engel O, Volkmer BG, JuarezBrito MA, Eggel M, Gschwend JE, Rubin MA and Day ML: Assessment of a fragment of E-cadherin as a serum biomarker with predictive value for prostate cancer. Br J Cancer 92: 2018-2023, 2005.

17. Kuefer R, Hofer MD, Gschwend JE, Pienta KJ, Sanda MG, Chinnaiyan AM, Rubin MA and Day ML: The role of an $80 \mathrm{kDa}$ fragment of E-cadherin in the metastatic progression of prostate cancer. Clin Cancer Res 9: 6447-6452, 2003.

18. Ahmed MI, Abd-Elmotelib F, Farag RM, Ziada NA and Khalifa A: Evaluation of some tissue and serum biomarkers in prostatic carcinoma among Egyptian males. Clin Biochem 32: 439-445, 1999.

19. Symowicz J, Adley BP, Gleason KJ, Johnson JJ, Ghosh S, Fishman DA, Hudson LG and Stack MS: Engagement of collagen-binding integrins promotes matrix metalloproteinase9-dependent E-cadherin ectodomain shedding in ovarian carcinoma cells. Cancer Res 67: 2030-2039, 2007.

20. Neurath MF and Finotto S: IL-6 signaling in autoimmunity, chronic inflammation and inflammation-associated cancer. Cytokine Growth Factor Rev 22: 83-89, 2011.

21. Alcover J, Filella X, Luqué P, Molina R, Izquierdo L, Augé JM and Alcaraz A: Prognostic value of IL-6 in localized prostatic cancer. Anticancer Res 30: 4369-4372, 2010.

22. Shariat SF, Kattan MW, TraxelE, Andrews B, Zhu K, Wheeler TM and Slawin KM: Association of pre- and postoperative plasma levels of transforming growth factor beta(1) and interleukin 6 and its soluble receptor with prostate cancer progression. Clin Cancer Res 10: 1992-1999, 2004.

23. Tumminello FM, Badalamenti G, Incorvaia L, Fulfaro F, D'Amico C and Leto G: Serum interleukin-6 in patients with metastatic bone disease: correlation with cystatin C. Med Oncol 26: 10-15, 2009.

24. Svatek RS, Jeldres C, Karakiewicz PI, Suardi N, Walz J, Roehrborn CG, Montorsi F, Slawin KM and Shariat SF: Pre-treatment biomarker levels improve the accuracy of post-prostatectomy nomogram for prediction of biochemical recurrence. Prostate 69: 886-894, 2009.

25. Pedrazzani C, Caruso S, Corso G, Marrelli D, Neri A, Berardi A and Roviello F: Influence of age on soluble E-cadherin serum levels prevents its utility as a disease marker in gastric cancer patients. Scand J Gastroenterol 43: 765-766, 2008.

26. Carroll JE, Low CA, Prather AA, Cohen S, Fury JM, Ross DC and Marsland AL: Negative affective responses to a speech task predict changes in interleukin (IL)-6. Brain Behav Immun 25: 232-238, 2011.

27. Sothern RB, Roitman-Johnson B, Kanabrocki EL, Yager JG, Roodell MM, Weatherbee JA, Young MR, Nemchausky BM and Scheving LE: Circadian characteristics of circulating interleukin-6 in men. J Allergy Clin Immunol 95: 1029-1035, 1995.

28. Katayama M, Hirai S, Kamihagi K, Nakagawa K, Yasumoto M and Kato I: Soluble E-cadherin fragments increased in circulation of cancer patients. Br J Cancer 69: 580-585, 1994.

29. Jurczok A, Zacharias M, Wagner S, Hamza A and Fornara P: Prospective non-randomized evaluation of four mediators of the systemic response after extraperitoneal laparoscopic and open retropubic radical prostatectomy. BJU Int 99: 1461-1466, 2007.

30. Lin MT, Yeh SL, Wu MS, Lin JT, Lee PH, Liaw KY, Chang KJ and Chen WJ: Impact of surgery on local and systemic responses of cytokines and adhesion molecules. Hepatogastroenterology 56: 1341-1345, 2009. 